\title{
The effect of action on perceptual feature binding
}

\author{
Inci Ayhan ${ }^{\mathrm{a}, \mathrm{b}, *}$, Melisa Kurtcan ${ }^{\mathrm{b}}$, Lucas Thorpe ${ }^{\mathrm{b}, \mathrm{c}}$ \\ a Department of Psychology, Bogazici University, Istanbul, Turkey \\ ${ }^{\mathrm{b}}$ Cognitive Science Program, Bogazici University, Istanbul, Turkey \\ ${ }^{\mathrm{c}}$ Department of Philosophy, Bogazici University, Istanbul, Turkey
}

\section{A R T I C L E I N F O}

\section{Keywords:}

Visuo-motor recalibration

Perceptual feature binding

Prediction

\begin{abstract}
A B S T R A C T
Color-motion asynchrony (CMA) refers to an apparent lag of direction of motion when a dynamic stimulus changes both color and direction at the same time. The subjective order of simultaneous events, however, is not only perceptual but also subject to illusions during voluntary actions. Self-initiated actions, for example, seem to precede their sensory outcomes following an adaptation to a delay between the action and the sensory feedback. Here, we demonstrate that the extent of the apparent asynchrony can be substantially reduced when direction change is induced by a voluntary key press following a delay adaptation regime. We also show that the reduced color-motion asynchrony effect size following a motor-sensory recalibration is not a result of a change in the onset of perceived direction change relative to that of the color. This is particularly important as it implies, for the first time in the literature, that voluntary action is not only important in forming action-sensory outcome associations but may also act as a binding factor between the two perceptual features of a sensory event.
\end{abstract}

\section{Introduction}

Visual processing is specialized, such that different features of a single object, i.e. color, motion, depth or spatial pattern, are encoded to some extent by dedicated systems linked to anatomically separate brain regions (Bartels \& Zeki, 1998; Livingstone \& Hubel, 1988; Shipp \& Zeki, 1985). How these modular patterns of activity are bound together to result in a single unified representation, however, is a question yet to be answered. According to the brain-time account, defended by Zeki and Bartels (1998), conscious temporal effects depend upon the temporal properties of the underlying brain processes. So, for example, the experienced order of events depends upon the temporal ordering of the underlying brain processes. Thus, they claim that the activity in a cortical area which is responsible for the processing of a particular feature, is not only associated with a sensory output but also generates an autonomous micro-consciousness for that specific attribute, for example activity in the motion area MT + generates the conscious perception of motion. In this account, perceptual binding is regarded as a post-conscious operation, which is directly influenced by the sensory processing latencies across the distributed modular processing systems running in an attribute-specific fashion. The idea that the temporal character of perceptual experience is reducible to the temporal properties of the sensory processors, however, has been challenged (Arnold \& Clifford, 2002; Bedell, Chung, Ogmen, \& Patel, 2003; Clifford, Spehar, \& Pearson, 2004; Nishida \& Johnston, 2002).
In their original study, Moutoussis and Zeki (1997) used a phenomenon called color-motion asynchrony (CMA), which is the perceptual difficulty of pairing the correct phases of color and motion direction of an oscillating pattern alternating between two states periodically at the same rate (e.g. green dots moving upwards and red dots moving downwards). Modulating the relative phases of color and direction of motion, Moutoussis and Zeki demonstrated that, compared to color, motion is perceived as delayed by about $78 \mathrm{~ms}$. They interpreted this perceptual asynchrony effect as evidence of different processing latencies for different features. CMA, however, was later shown to be stimulus-specific such that transparent surfaces of green and red dots (e.g. arranged in thin stripes) drifting in opposite directions of motion reduce the percept of asynchrony as opposed to those, where two phases of motion and color are presented sequentially as homogenous blocks (Clifford et al., 2004). To explain the effect, Clifford et al. suggested that when all dots oscillate in phase, responses in populations of neurons in the area MT + are subject to a neurophysiological effect called directional inhibition (Snowden, Treue, Erickson, \& Andersen, 1991) which slows down the formation of a new motion-defined surface representation, thus, introducing a temporal delay into the feedback signal sent into the lower-level motion-processing areas (e.g. area V1). In the case of motion transparency, on the other hand, motion representation is maintained for both transparent motion-defined surfaces, resulting in a temporally accurate binding of color and motion. Thus, Clifford, Spehar and Pearson explain CMA within the context of a

\footnotetext{
* Corresponding author.
} 
reflexive feedback mechanism from higher-level motion-integrating areas (e.g. MT+) into those, where local motion and color are processed at higher spatial resolutions.

Area MT + has been identified as interconnected with the ventral intraparietal area (Maunsell \& van Essen, 1983; Ungerleider \& Desimone, 1986) which is known to contain both visual and somatosensory neurons (Colby, Duhamel, \& Goldberg, 1989; Duhamel, Colby, \& Goldberg, 1989). At the populational level, neurons in the area MT+ have also been demonstrated to show a task-related co-activity with those in the primary motor cortex (Kruse, Dannenberg, Kleiser, \& Hoffmann, 2002) which has been proposed to play a role in the actionsensory outcome prediction (Hughes \& Waszak, 2011). Here, we study the effects of voluntary causal action on the temporal dynamics of feature binding. If CMA is in fact caused by a temporal delay in the formation of surface representations at the level of area MT + , then, forming a predictive action-sensory outcome association between a selfinitiated key press and a sensory change in a CMA paradigm could reduce the asynchrony in the temporal binding of color and motion.

To test this hypothesis, we integrated a behavioral motor actionsensory outcome association paradigm (Stetson, Cui, Montague, \& Eagleman, 2006) into a single motion-direction-change CMA task (Linares \& López-Moliner, 2006) and demonstrated -for the first timethat action not only exerts influence over the action-sensory outcome association but also modulates the perceptual feature binding of concurrent visual events.

\section{General methods}

\subsection{Participants}

Participants were five naïve participants and the two authors in Experiments 1 and $2(\mathrm{~N}=7)$, and three naïve participants and the two authors in Experiment $3(\mathrm{~N}=5)$. Sample size was chosen according to the previous studies, which conducted a psychophysical investigation of color-motion asynchrony using 2-4 participants (Arnold \& Clifford, 2002; Bedell et al., 2003; Linares \& López-Moliner, 2006). All participants had normal or corrected-to-normal vision via glasses or lenses with no color vision deficit. All experiments were compliant with the Bogazici University research ethics requirements, as well as the Declaration of Helsinki. Participants gave their consent prior to experiments.

\subsection{Stimuli and materials}

Experiments were run in a Samsung CRT monitor with a refresh rate of $85 \mathrm{~Hz}$. Display was calibrated using a Datacolor Spyder4 Elite colorimeter. Maximum and minimum luminance were set to 138.6 and $4.09 \mathrm{~cd} / \mathrm{m}^{2}$, respectively. Stimuli were coded via Matlab Psychophysics Toolbox. Participants were seated approximately $57 \mathrm{~cm}$ away from the screen with a spatial resolution of $1280 \times 1024$ pixels. From this distance, 35 pixels corresponded to $1^{\circ}$ of visual angle. The stimuli set consisted of $600-800$ dots (diameter size: $0.3^{\circ}$ ) distributed randomly across the screen around an aperture (diameter size: $4.3^{\circ}$ ), in the middle of which was presented a fixation spot. The action was recorded via a standard keyboard. On a black background, dots were all moving uniformly at a speed of $6^{\circ} / \mathrm{s}$. For the calibration phase and the visuo-motor temporal ordering task, dots were presented in a neutral grey. In the color-motion asynchrony correspondence task, we used individual equiluminant magenta and cyan values that were derived from a minimum-motion experiment (Cavanagh, MacLeod, \& Anstis, 1987) for each participant, individually.

\subsection{Procedure}

Experiments were conducted in a quiet and dark cubicle situated at Bogazici University Vision Lab. Participants were requested to maintain their visual fixation on a cross presented at the center of display. Head position was fixed by a chin rest. All experiments were conducted using a two-alternative forced choice (2-AFC) paradigm. In each experiment, there were both a baseline and a delayed feedback conditions, where participants were presented with either a non-delayed or a delayed sensory feedback as result of their self-initiated action. Order of the delay and no delay blocks were randomized across participants in all experiments. Data were fitted a Cumulative Gaussian function. The fitting method was the "Maximum Likelihood (MLH)" method described by Watson (1979).

\subsubsection{Experiment 1: motor-sensory recalibration effect on a second-order visual feature change}

Stetson et al. (2006) have shown that the temporal order of a sensory outcome and a voluntary motor action is perceived as reversed following an adaptation phase, where the sensory feedback is consistently presented after a delay following a manual response. In order to study this effect in a paradigm with a second-order visual feature change, such as in the motion direction, as opposed to a first-order change, such as in the appearance of a signal dot, as was used in the original setup, we examined the extent of motor-sensory recalibration as a function of different temporal delays between the self-initiated action and the sensory feedback during the calibration phase: (i) $0 \mathrm{~ms}$ for the baseline and (ii) $105.9 \mathrm{~ms}$ for the experimental condition. Observing a temporal recalibration between the perceived timing of action and that of a motion direction change would support the conclusion that a motor-sensory recalibration, previously shown between an action and a first-order sensory change (e.g. the appearance of a dot), could also be established between an action and a second-order sensory change (e.g. change in the direction of motion).

2.3.1.1. Procedure. We used a temporal order judgment (TOJ) paradigm, which was composed of a recalibration (Fig. 1a) and a test phase (Fig. 1b). In the recalibration phase (5-12 trials), the aim was to adapt participants to a constant delay between a key press and a sensory feedback. In the test trials, the delay was eliminated, thus, the sensory outcome was always concurrent with the key press. Each trial started with the presentation of an array of grey dots drifting along a certain motion trajectory (either upwards or downwards), where participants were asked to press a key on a keyboard at a time of their choice within the limits of a fixed interval $(600 \mathrm{~ms})$. The key press would then trigger a $180^{\circ}$ change in the direction of motion of the dot array after a fixed delay. In the experimental blocks the delay was kept at $105.9 \mathrm{~ms}$. In the baseline condition there was no delay. The trials in which participants failed to press the key within the time limit were repeated. Following the recalibration phase, the fixation spot changed color from grey to yellow to signal the test trial and participants were expected to make temporal order judgments. Participants were clearly instructed that this cue is not a signal to be acted upon, but rather to get prepared for the incoming test trial, where they would make a selfinitiated motor act on a time point they choose within a limited interval. They were also instructed to press the key at a time which would be as variable as possible within the given limited range. The temporal order between the key press and the sensory feedback was randomized so that the key press could either be followed or preceded by a change in the motion direction of the dot array. Averaging participant's reaction times across trials, an algorithm probabilistically computed the time at which the direction change would occur by assigning a random value from a uniform distribution within the range of the mean reaction time $-/+275$ ms. The delay between the two events was varied across different trials and participants were asked to report whether their own key press or the change in the motion direction of the stimulus array occurred first, by using the arrow keys on a keyboard. The average number of trials was around 140-147 trials. 
a

RECALIBRATION TRIALS (\# 5 - 12)

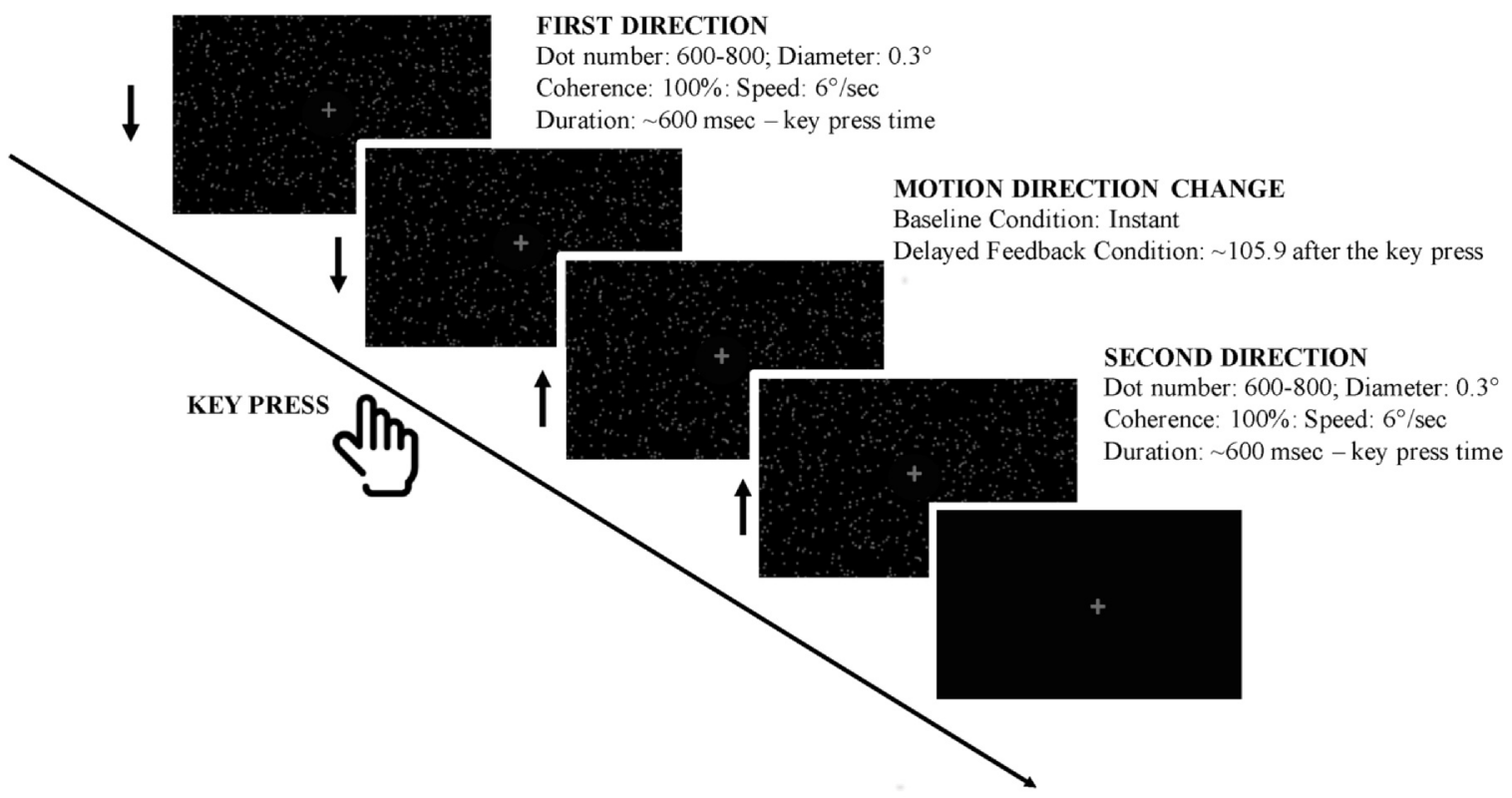

b

TEST TRIAL

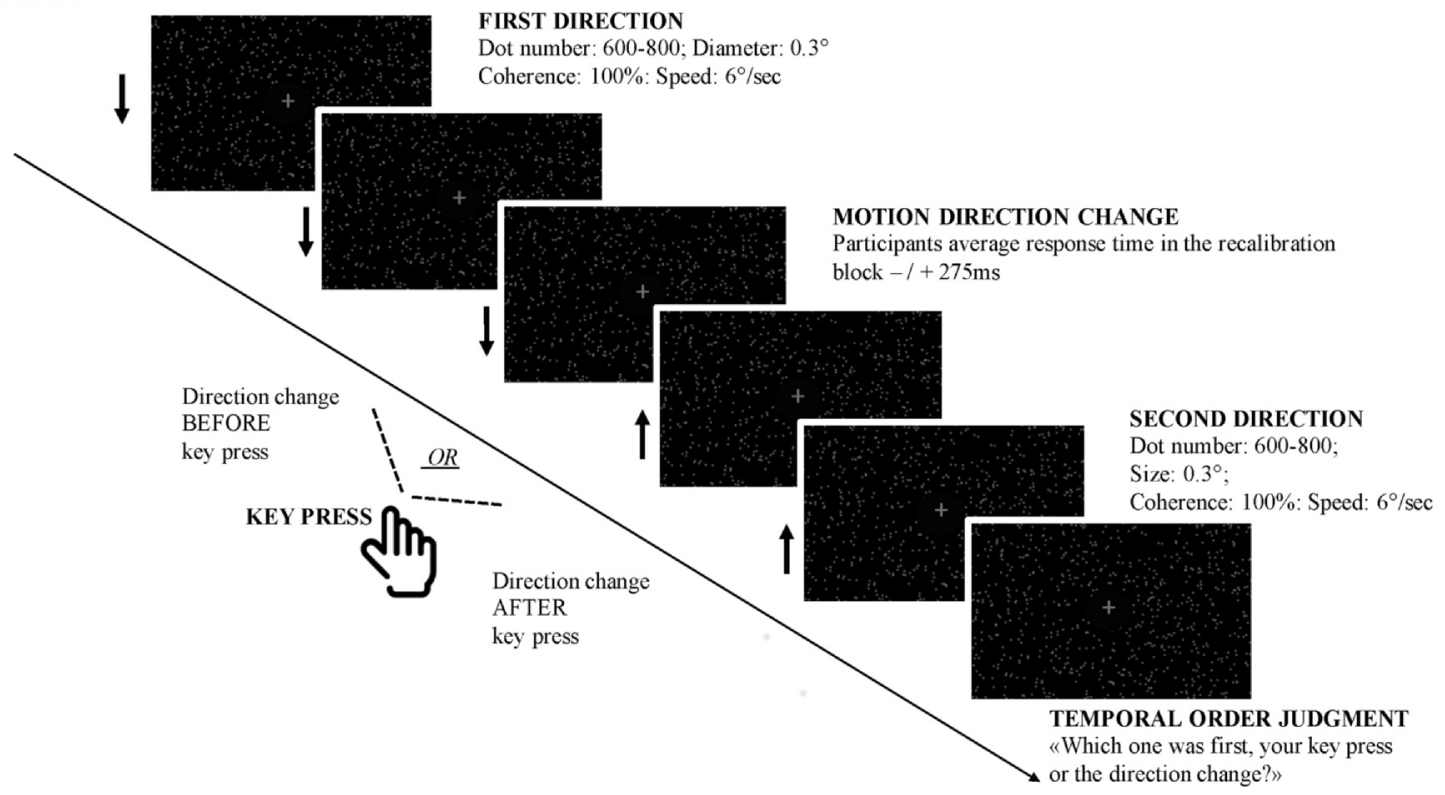

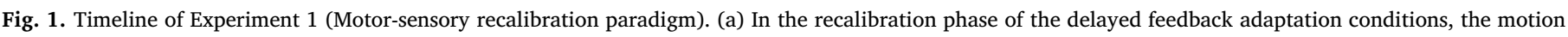

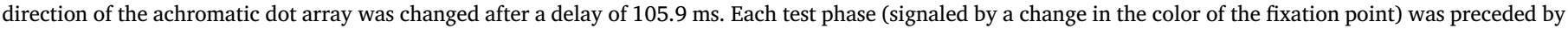

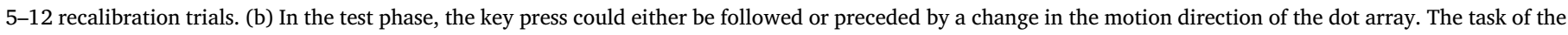

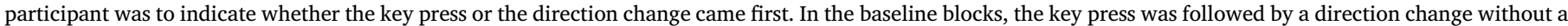
delay in the recalibration trials.

2.3.1.2. Results. Behavioral data was plotted as individual psychometric functions per condition, where the relative time between the key press and the direction change were on the $x$-axis and "proportion of direction change before the key press" on the $y$-axis. The Point of Subjective Simultaneity (PSS) values indicated the $50 \%$ point on the psychometric function. Whereas negative values indicated the trials where participant performed the key press before the change in the visual motion direction, positive values indicated the opposite, namely, the trials where participant performed the key press after the change in the visual motion direction. Thus, the distribution of responses was centered around a PSS value of zero for an unbiased observer. Examples of individual distributions of the relative time between the 
a

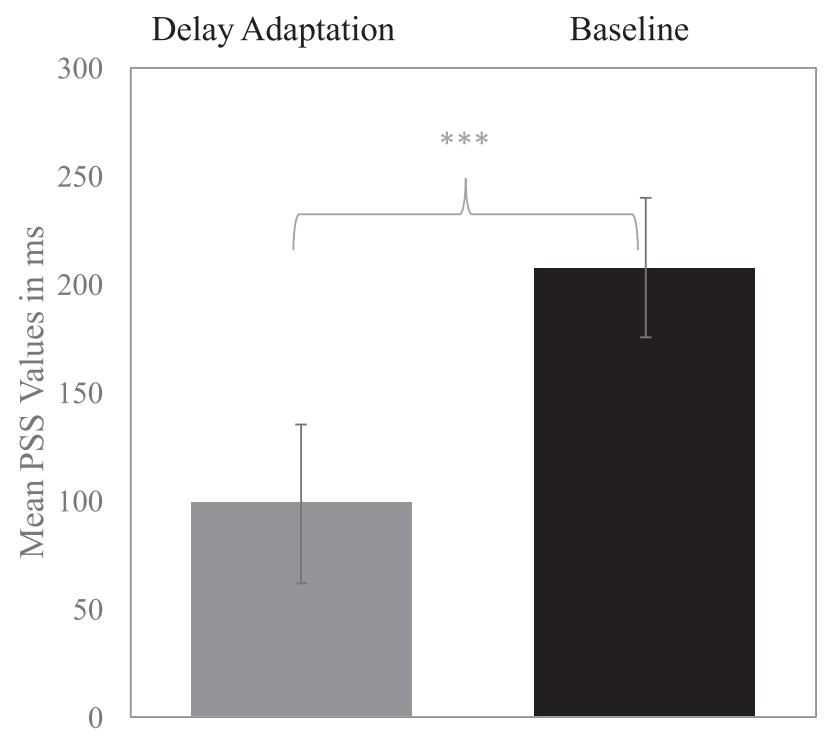

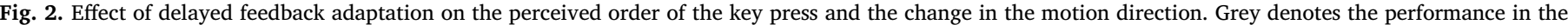

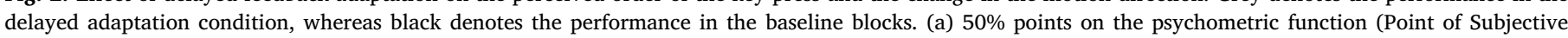

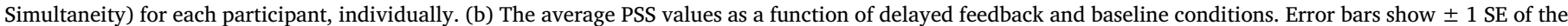
mean.

direction change and the keypress across different trials in the delayed and baseline conditions may be found in the Supplementary Materials (Supp. Fig. 1A).

A two-tailed paired sample $t$-test on individual data (Fig. 2a) indicated that the mean scores were significantly lower in the delayed feedback condition $(M=207.4, S D=86.36)$ than in the baseline $(\mathrm{M}=98.3, \mathrm{SD}=96.06), t(6)=-5.82, \mathrm{p}=.001, \eta^{2}=0.485$ (Fig. 2b). Individual data provided evidence for a strong bias towards the "key press before the direction change" responses, supporting the earlier findings of Stetson et al. Note that the size of the recalibration in our experiment is larger than that in the Stetson et al. study, which demonstrates that using a second order change (e.g. change in the direction of motion), rather than a first order change (e.g. change in the color) increases the effect size of the visuo-motor recalibration. In this connection, recent evidence has shown that there is a temporal asymmetry in the recalibration of the perceived simultaneity following a systematic training to leading or lagging stimuli such that the perceived timing of lagging stimuli is changed more strongly than that of the leading stimuli (Rohde, Greiner, \& Ernst, 2014; Yarrow, SverdrupStueland, Roseboom, \& Arnold, 2013). Rohde et al. (2014) linked this asymmetry to the effect of sense of agency on the visuo-motor temporal integration. In this context, what we can speculate with regards to why we might have observed a larger effect size can be the sense of agency acting upon the visuo-motor temporal integration within a larger temporal window of recalibration in the case of a second-order sensory outcome.

Our current results imply that the sensory-motor recalibration effect can be induced for a second-order single-feature-change (i.e. motion direction) paradigm, as well as a first-order single-feature-change (i.e. light flash) paradigm. This finding supports the earlier findings of Heron, Hanson, and Whitaker (2009), where they had demonstrated using different sensorimotor pairings (e.g., adapt 'mousepress- flash', test 'mousepress-click') that the magnitude of motor-sensory recalibration is the same even when used different sensory modalities during the adaptation and test trials. Similarly, Sugano, Keetels, and Vroomen (2010) have also demonstrated that the shift in the Point of Subjective Simultaneity across delay adaptation and baseline conditions was similar in both within-modal, where the modality of the outcome stimulus was the same as the adapted one, and cross-modal conditions, where the modality of the outcome stimulus was different than the adapted one. Together with our current results, these may suggest that motor-sensory association is pretty flexible and transferrable across within- and between-modality features, possibly as long as the causality relationship is preserved (Heron et al., 2009).

\subsubsection{Experiment 2A: visuo-motor feature-correspondence task}

It has been claimed that CMA is caused by a delay in forming a new surface representation at the area MT + (Arnold \& Clifford, 2002; Bedell et al., 2003; Clifford et al., 2004, 2010) due to the physiologically reported effects of opposite-motion inhibition (Snowden et al., 1991). If this were the case, then, visuo-motor recalibration could potentially establish an action-outcome prediction and reduce the delay in the extra striate motion-processing areas. Thus, our prediction was that adaptation to delayed sensory feedback to the self-caused action would decrease the size of the asynchrony between color and motion in a visuo-motor test paradigm.

2.3.2.1. Procedure. As in Experiment 1, each experimental block consisted of both recalibration (Fig. 1a) and test trials (Fig. 3a). In the recalibration phase, participants were adapted to a constant delay between their self-initiated key press and the change in the motion direction of dots in the stimulus array. In the test trials, the delay was removed. In order to avoid color adaptation, the adaptation phase was conducted using grey dots on a black background. In the test trials, which were signaled by a transient change in the color of the fixation spot, participants were asked to make a key press to change the direction of an array of equiluminant chromatic dots alternating between two states, i.e. magenta and cyan, drifting upwards or 


\section{TEST TRIAL}

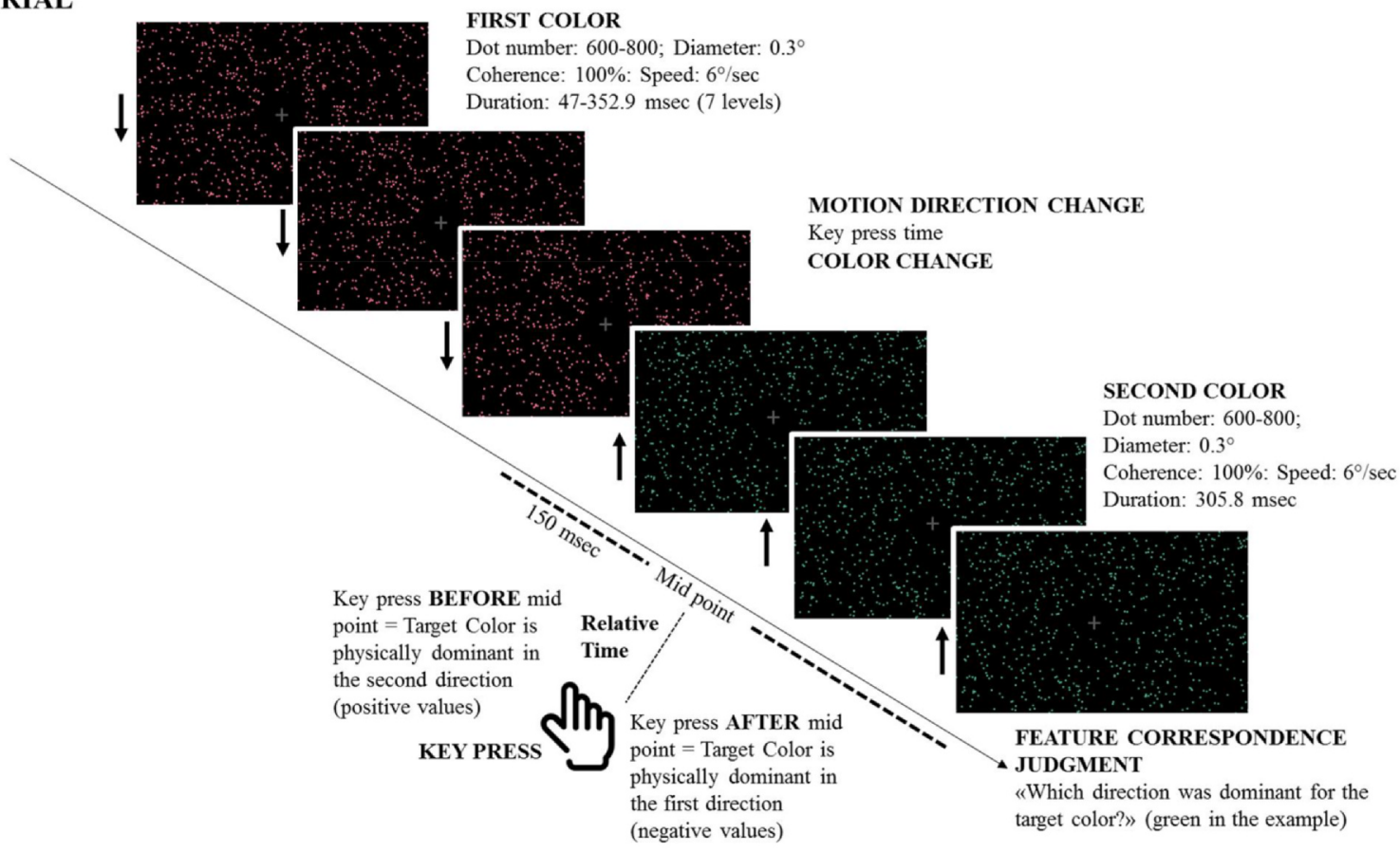

b

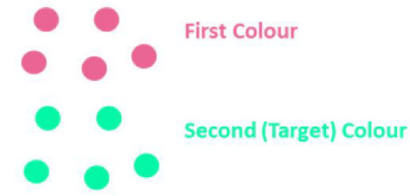

Task: Which is the dominant direction for the target color: Up or Down? Experiment 2A: Keypress during the test trials.

Experiment 2B: NO keypress during the test trials.

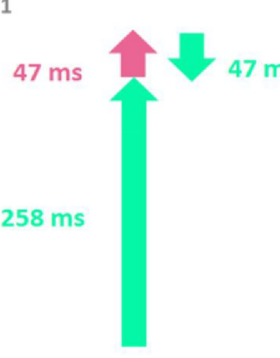

\section{1}

$105.88 \mathrm{~ms}$

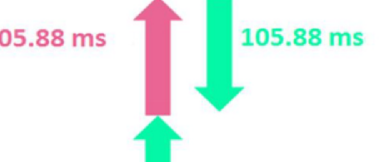

$200 \mathrm{~ms}$
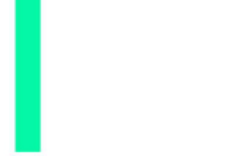

3
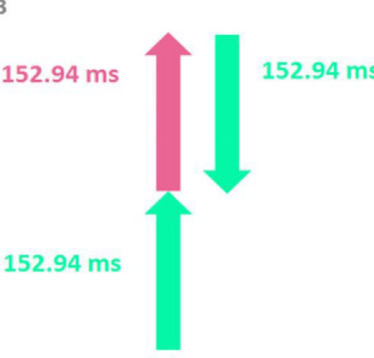

$105.88 \mathrm{msec}$

$47 \mathrm{~ms}$

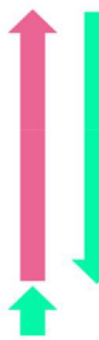

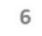

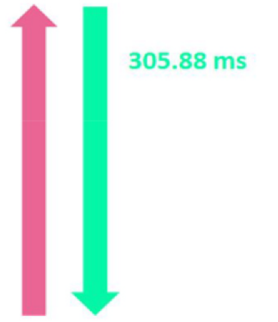

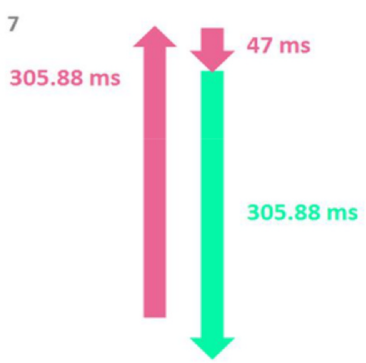

- The first color interval varies between $47-352.9 \mathrm{~ms}$ across 7 levels.

- The second interval which is the target color interval is always kept at $\mathbf{3 0 5 . 8 8} \mathbf{m s}$.

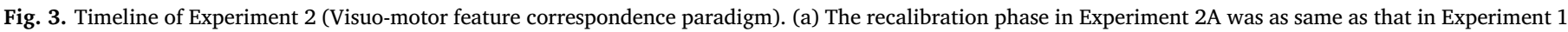

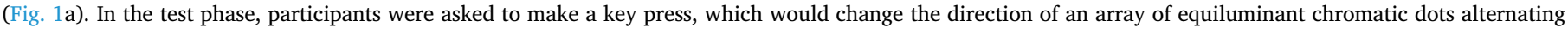

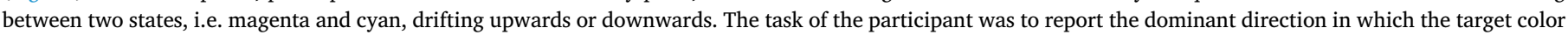

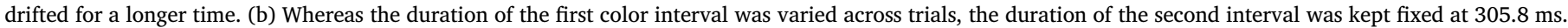

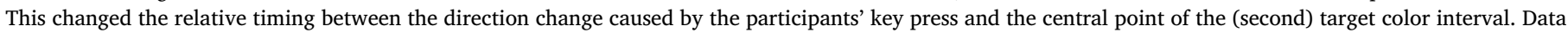

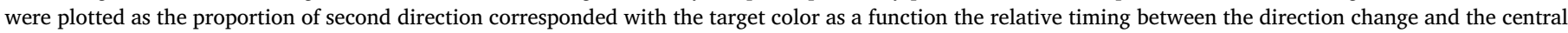
point of the target color interval. (For interpretation of the references to color in this figure legend, the reader is referred to the web version of this article.) 
downwards. Participants were then instructed to judge the dominant direction associated with the dots in target color. That is, they judged whether they perceived the dots with the target color drifting mostly in the first or the second direction of motion.

Note that target color was always the one that was presented at the end of the trial as the second color. In different test trials, we manipulated the duration of the first color interval (using 7 levels: 47, 105.9, 152.9, 200, 258.8, 305.8, 352.9 ms) but kept fixed the second interval (which was the target color) at $305.8 \mathrm{~ms}$. Different durations of the first color interval were randomized across trials. This changed the relative timing between the direction change caused by the participants' key press and the central point of the target color interval (Fig. 3b). Examples of individual distributions of the relative time between the direction change and the midpoint of the target color across different trials in the delayed and baseline conditions may be found in the Supplementary Materials (Supp. Fig. 1B). We used individual equiluminant magenta and cyan values that were derived from a minimum-motion experiment (Cavanagh et al., 1987) using the DKL color space (Derrington, Krauskopf, \& Lennie, 1984) for each participant. Each experimental block consisted of 49 trials, making a total of

2.3.2.2. Results. To analyze data, we followed a procedure similar to that used by Linares and López-Moliner (2006). The data was plotted as a distribution of the proportion of trials in which the second direction (the direction causally preceded by the participants' key press) corresponded with the target color as a function of the relative timing

a

Experiment 2A: Visuo - Motor Feature Correspondence

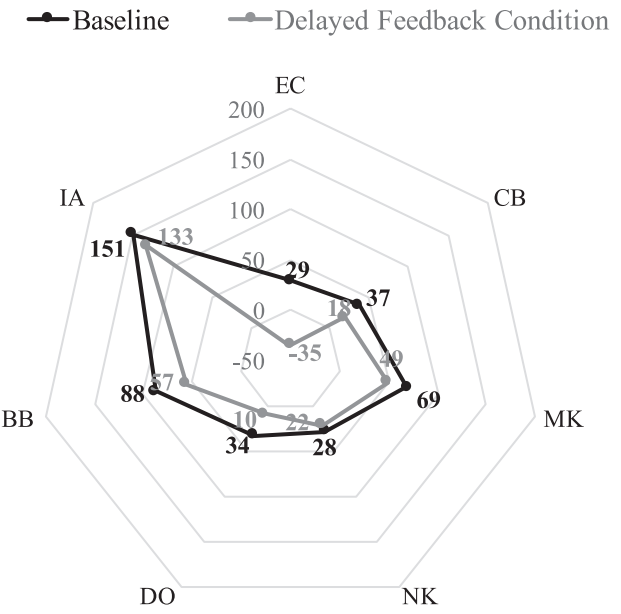

(PSE values in milliseconds) 147 trials for 3 blocks in a condition.

between the direction change and the center of the target color interval (Fig. 4c). Negative values of the relative timing denoted the trials, where the key press had occurred after the first half $(152.9 \mathrm{~ms})$ of the midpoint of the target color interval $(305.8 \mathrm{~ms})$. In these trials, dots with the target color were mostly presented in the first direction of motion. If the participants pressed the key at the midpoint of the target color, then the target color would be presented moving through both directions for equal amounts of time, which would correspond to a relative timing of zero. Positive values, on the other hand, would represent the conditions in which the key press occurred before the midpoint of the target color interval, that is either during the first color or the first half of the target color. In this case, dots with the target color moved mostly through the second direction. The data was fitted a cumulative Gaussian function in order to obtain the Point of Subjective Equality (PSE) as a measure of the perceptual asynchrony between color and motion direction. Due to CMA, in the baseline condition, the PSE of the distribution was shifted towards the positive values (Fig. 4c, black function) representing the conditions in which the target dots were presented in the second direction of motion for longer, which corresponds to a perceptual delay of direction change (Linares \& LópezMoliner, 2006). We then compared the PSE's across delayed and baseline conditions and observed a significant reduction in CMA $(\sim 26 \mathrm{~ms})$ following a delayed feedback adaptation (Fig. 4c, grey function). A paired sample $t$-test on individual data points (Fig. 4a) demonstrated that the PSE scores were significantly lower in the delayed feedback condition ( $M=36.22$, $S D=52.2$ ) than in the baseline condition $(\mathrm{M}=62.22, \mathrm{SD}=45.25), t(6)=-3.74, \mathrm{p}=.01$,
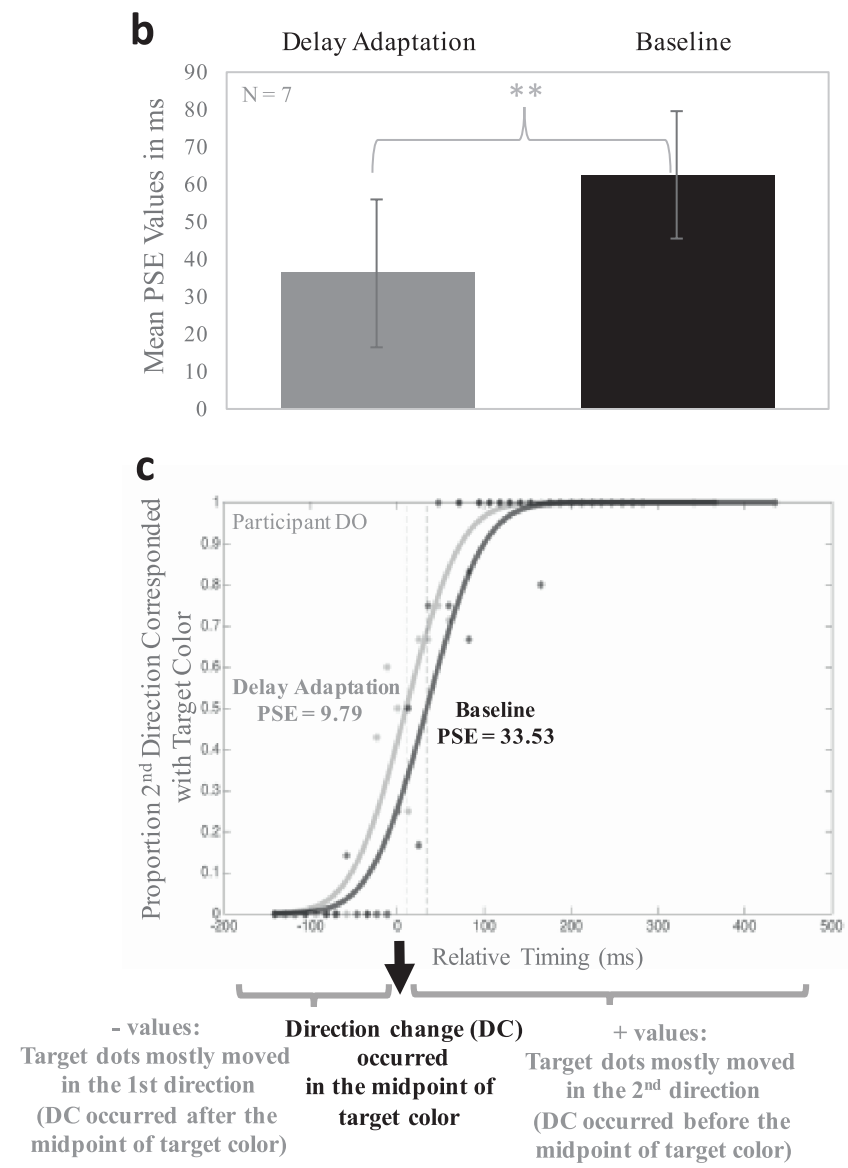

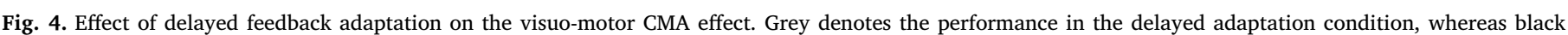

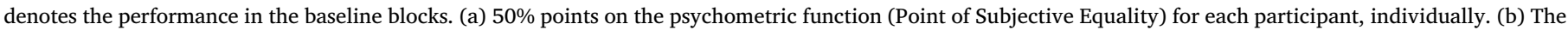

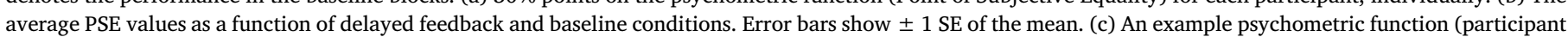

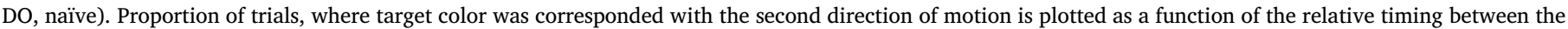
direction change and the midpoint of the target color. 
$\eta^{2}=0.28$ (Fig. 4b). In fact, a one-sample $t$-test demonstrated that whereas the average PSE values were significantly different than a reference value of $0 \mathrm{~ms}$ in the baseline condition, $t(6)=3.64, \mathrm{p}=.005$, $\eta^{2}=0.69$ (one-tailed), the significance, and thus, the color-motion asynchrony, disappeared in the delay adaptation condition, $t$ (6) $=1.84, p=.058, \eta^{2}=0.36$ (one-tailed) following a delayed sensory feedback adaptation in response to a self-caused action. Results also demonstrated that motor-sensory adaptation formed between a key press and the motion direction change of an achromatic array of dots was transferred to a stimulus composed of multiple features (i.e. chromatic dots in motion).

As well as the PSE analysis, we also conducted an analysis on the discrimination threshold, defined as the difference between the $50 \%$ and $84 \%$ point on the psychometric function. A paired-sample $t$-test indicated that the discrimination threshold is not statistically different in the delayed feedback condition $(M=58.09, S D=24.13)$ than in the baseline condition $(M=50.76, S D=16.93), t(6)=0.69, p=.51$, $\eta^{2}=0.07$, suggesting no change in task difficulty.

In our visuo-motor CMA paradigm, change in the direction of motion was contingent upon the participant's keypress. In other words, the relative timing of the direction change with respect to the midpoint of the target array was variable in each trial. Thus, it was important to check whether the keypress time (indexed as the average relative timing) was correlated with the magnitude of the visuo-motor CMA effect, which would indicate a response bias. A correlation analysis between the average relative timing (Mean $=105.86, \mathrm{SD}=49.59$ ) and average visuo-motor CMA effect (Mean $=-25.99$, SD $=18.38$ ), however, indicated that these two variables do not correlate, $r$ (5) $=0.15, p=.74, r^{2}=0.02$ (Fig. 6a), implying that the shift we observed in the delayed feedback adaptation block was rather a genuine visuo-motor effect.

\subsubsection{Experiment 2B: visual feature-correspondence task}

In Experiment 2A, participants were asked to make a self-initiated action during both the calibration and the test phase. Therefore, the reduction in the effect size of the CMA could be due to a mere visual adaptation that took place in the calibration phase irrespective of a causal link constructed between the motor action and the sensory feedback. In order to exclude the possibility that the decrease in the size of CMA is not a consequence of self-initiated action but rather a visual adaptation, we conducted a further experiment, where participants generated action during the recalibration phase but not in the test trials.

2.3.3.1. Procedure. In Experiment 2B, we conducted a visual featurecorrespondence task preceded by the visuo-motor calibration trials. In contrast to the previous experiments, in the test trials of Experiment 2B, the participants passively observed the stimuli, and did not have to press a key. The task was to judge the dominant direction for the target color. As in Experiment 2A, we manipulated the relative timing between the direction change and the color change by varying the first color interval between 47 and 352.9 ms across 7 levels. Each experimental block consisted of 49 trials, making a total of 147 trials for 3 blocks in a condition. Trials in which participants made a key press in the test trial were omitted from the analysis.

2.3.3.2. Results. Using the same method of analysis as we applied in Experiment 2A, we compared the PSE's across delayed and baseline conditions. A paired sample $t$-test on the individual data (Fig. 5a) indicated that there is no significant difference between the PSE scores in the delayed feedback condition $(\mathrm{M}=35.30, \mathrm{SD}=48.14)$ and in the baseline $(\mathrm{M}=36.20, \mathrm{SD}=45.45), t(6)=-0.33, \mathrm{p}=.76, \eta^{2}=0.003$ (Fig. 5b, column 3). Remember that positive values represent the conditions in which the key press occurred before the midpoint of the target color interval, that is either during the first color or the first half of the target color. In this case, dots with the target color moved mostly through the second direction. Thus a bias of $35.30 \mathrm{~ms}$ in the delayed and $36.20 \mathrm{~ms}$ in the baseline conditions indicates a color-motion asynchrony effect. Although this is a briefer delay than the original $73 \mathrm{~ms}$ average delay reported by Linares and López-Moliner (2006) in a color-motion asynchrony paradigm with a single change, a one-sample $t$-test demonstrated that the average PSE values in Experiment 2B were significantly different than a reference value of $0 \mathrm{~ms}$, both in the delayed, $t(6)=1.94, p=.05, \eta^{2}=0.385$ (one-tailed) and in the baseline conditions, $t(6)=2.11, p=.04, \eta^{2}=0.426$ (one-tailed), providing evidence that we also demonstrated color-motion asynchrony in a single change paradigm where there is no key press.

That the size of CMA did not get reduced following a visuo-motor delay adaptation paradigm, where there is no self-initiated action during the test trials implies that the reduction in CMA following the motor-sensory adaptation observed in Experiment $2 \mathrm{~A}$ is attributable to the voluntary action-sensory outcome pairing. Fig. 5b summarizes the results of Experiment 1 and 2, where the difference scores between the delayed feedback and the baseline conditions in the visuo-motor feature correspondence task $(\mathrm{M}=-25.99, \mathrm{SD}=18.38)$ is significantly larger than those in the visual feature correspondence task $(\mathrm{M}=-0.90$, $\mathrm{SD}=7.24), t(6)=-4.26, \mathrm{p}=.005, \eta^{2}=0.335$.

A paired-sample $t$-test indicated that the function widths are not statistically different between the delayed feedback condition $(\mathrm{M}=42.50, \mathrm{SD}=16.19)$ and the baseline condition $(\mathrm{M}=50.94$, $\mathrm{SD}=20.27), t(6)=-0.94, \mathrm{p}=.39, \eta^{2}=0.13$, suggesting no change in task difficulty.

\subsubsection{Experiment 3: visual temporal ordering task in the presence of self- initiated action}

Bedell et al. (2003) demonstrated that the size of CMA depends on the nature of the task. For example, the asynchrony is larger in the feature correspondence task, where participants reported which color (green or red) was more dominant during the target dot motion than in the temporal ordering task, where participants reported whether the change in the dot color occurred before or after the onset and offset of motion. These results indicated that the asynchrony cannot be explained within the context of a brain-time account. Such an account explains the asynchrony between the conscious experience of change of direction and that of color, in terms of the neural delays in the processing of motion with respect to color in the visual brain areas MT and V4, respectively (Moutoussis \& Zeki, 1997). In order to see whether adaptation to a delayed sensory feedback to voluntary action reduced the CMA by shifting the perceived temporal onset of the motion direction change relative to the color change at an earlier point in the timeline in our current paradigm, we conducted a control experiment, where participants were tested on a visual temporal ordering task in the presence of a voluntary action. If the motor-sensory recalibration acts on CMA by binding the event onset of the second-order change and the action, thus, changing the temporal position of the direction change, then, we would expect to see a shift in the point of subjective simultaneity between the color and the direction change in a temporal order judgment (TOJ) task.

2.3.4.1. Procedure. Using a similar stimulus set to the previous experiment (changing the first color interval between 47 and $352.9 \mathrm{~ms}$ across 7 levels) we conducted a visual temporal ordering task following visuo-motor recalibration trials. Participants were required to judge the temporal order of motion direction and color change that is whether the direction change had taken place before or after the change in color. Each experimental block consisted of 49 trials, making a total of 147 trials for 3 blocks in a condition.

2.3.4.2. Results. We plotted the proportion of direction change reported to occur after color change as a function of the relative time between the direction change and the color change across different trials. Examples of individual distributions of the relative time between the direction change and the color change across different trials in the 
a

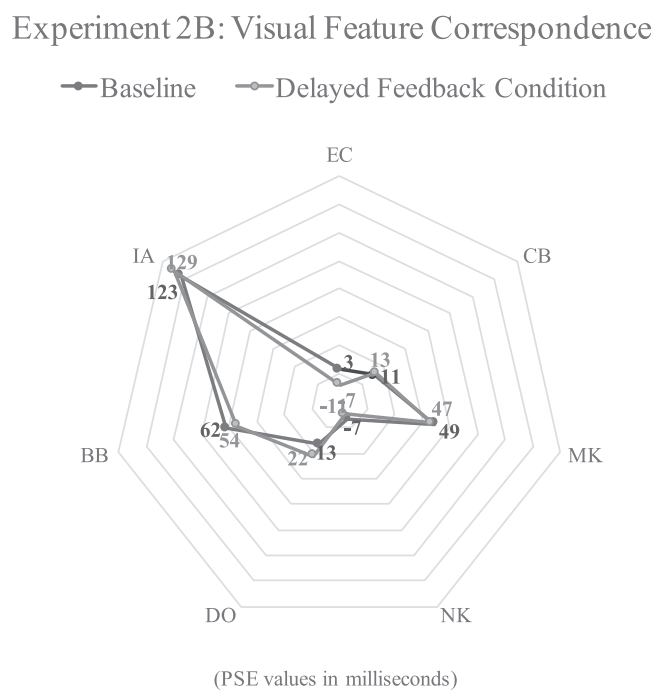

b

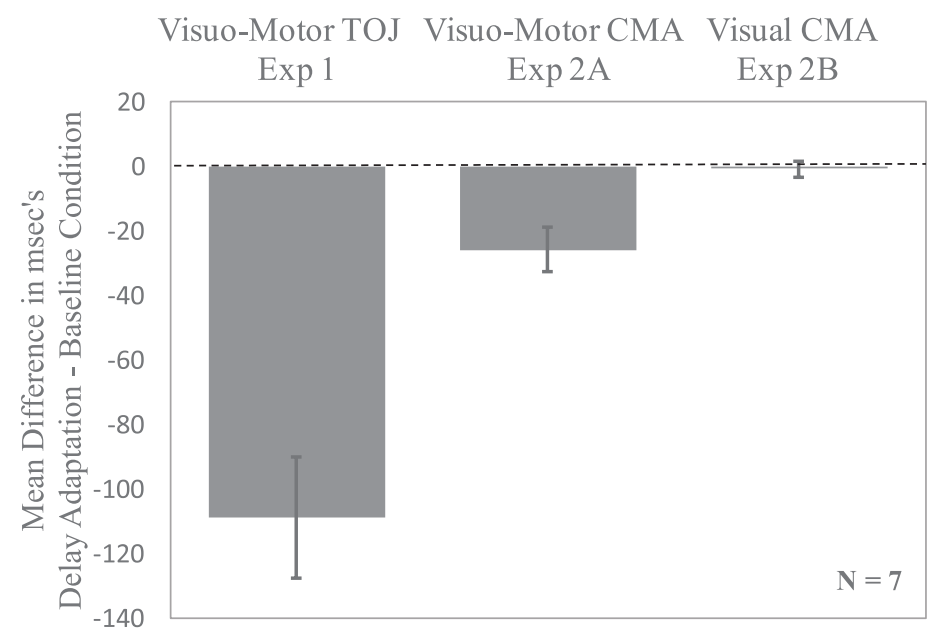

Fig. 5. Effect of delayed feedback adaptation on the visual CMA effect. Grey denotes the performance in the delayed adaptation condition, whereas black denotes the performance in the baseline blocks. (a) 50\% points on the psychometric function (Point of Subjective Equality) for each participant, individually. (b) On the $x$-axis are different experiments (Experiments 1 - Visuo-Motor Temporal Order Judgment, 2A - Visuo-Motor Color-Motion Asynchrony and 2B - Visual Color-Motion Asynchrony). On the $y$-axis are the average effects (performance in delayed feedback adaptation-performance in baseline blocks). Error bars show \pm 1 SE of the mean.

delayed and baseline conditions may be found in the Supplementary Materials (Supp. Fig. 1C). A paired sample $t$-test on individual data points indicated that there is not a significant difference between the PSE scores in the delayed feedback condition $(M=44.05, S D=66.25)$ than those in the baseline ( $M=50.65, S D=63.60), t(4)=-0.53$, $\mathrm{p}=.62, \eta 2=0.017$ (Fig. $6 \mathrm{~b}$, column 2). More interestingly, a paired samples $t$-test shows that the mean effect (Delay - No Delay) in Experiment $2 \mathrm{~A}(\mathrm{M}=31.29, \mathrm{SD}=19.10)$ is significantly greater than that in Experiment $3(\mathrm{M}=6.60, \mathrm{SD}=27.70), t(4)=3.99, \mathrm{p}=.016$, $\eta 2=0.799$, which may indicate that the results in Experiment $2 \mathrm{~A}$ cannot be explained by a binding between the onset of the voluntary action and the onset of the perceived visual motion direction change. Thus, the reduction in the CMA is most likely not a result of a shift in the perceived onset of the second-order change.

The positive values in this experiment represent the conditions in which the direction change occurred after the color change. Thus, for participants to report that direction and color change occurred at the same time, direction change had to lag color change for approximately 44.05 in the delayed adaptation and $50.65 \mathrm{~ms}$ in the baseline condition. This means that in contrary to the feature correspondence task, we a

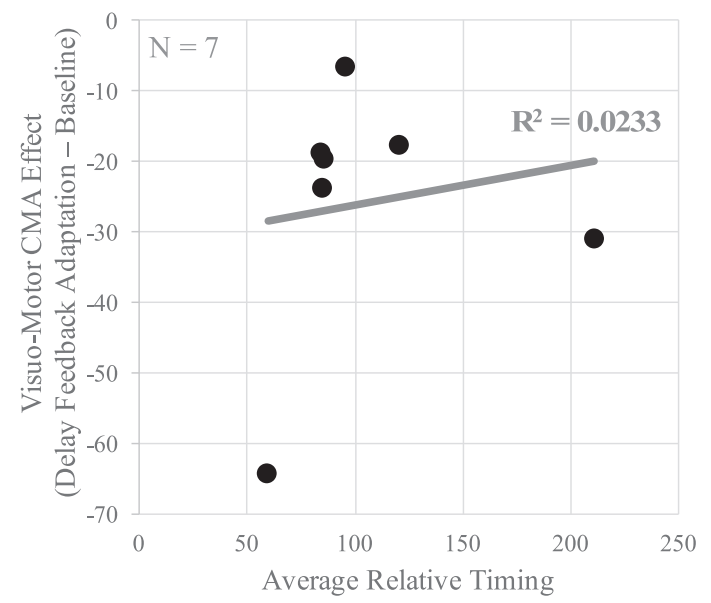

b

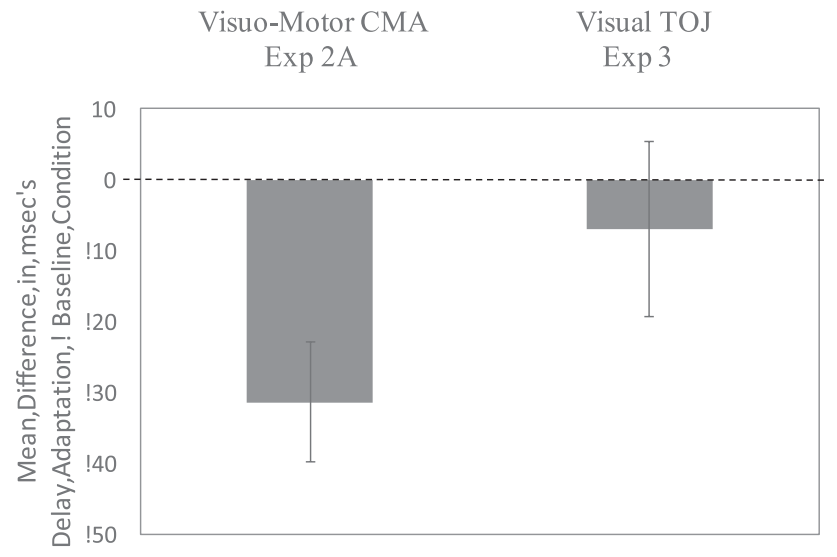

Fig. 6. Effect of delayed feedback adaptation on the perceived temporal order. (a) Visuo-motor CMA effect (performance in the delayed feedback adaptationperformance in the baseline blocks) is plotted as a function of the average relative timing of the direction change with respect to the midpoint of the target color interval. Each dot represents a participant's performance. The grey line is the linear fit of the data points. The adjusted coefficient of determination $\mathrm{R}^{2}$ is reported above the fitting line. (b) On the $x$-axis are different experiments (2A - Visuo-Motor Color-Motion Asynchrony and 3 - Visual Temporal Order Judgment). On the $y$ axis are the average effects (performance in delayed feedback adaptation-performance in baseline blocks). Error bars show \pm 1 SE of the mean. 
found motion change to be perceived earlier than color change in the temporal ordering task. Although this seems to be an interesting finding, one sample $t$-test demonstrated that this bias is not significantly different from the reference point of $0 \mathrm{~ms}$ neither in the delayed, $t$ (4) $=1.49, p=.106$ (one-tailed) nor in the baseline condition, $t$ (4) $=1.78, p=.075$ (one-tailed). Moreover, we noted that some participants in Bedell et al. have also shown a similar trend, particularly when the temporal order judgment was made between the onset of the motion change and color change. Thus, we may conclude that depending on the experimental task (e.g. temporal ordering) and in particular, the presence of an action-sensory outcome association as was the case in our paradigm, direction change may be perceived as preceding the color change.

A correlation analysis also indicated that the motor-sensory recalibration effect in Experiment 1 (Mean $=-109.15$, SD $=49.55$ ) does not correlate with the visuo-motor CMA in Experiment $2 \mathrm{~A}$ (Mean $=25.99, \mathrm{SD}=18.38), \mathrm{r}(5)=0.37, p=.41, \mathrm{r}^{2}=0.14$, which together demonstrate that the change in the perceived timing between the keypress and the direction change does not correlate with the strength of the visuo-motor CMA and that the effect is directly related to the feature correspondence rather than to the perceived onset time of the second-order change.

A paired-sample $t$-test indicated that the function widths are not statistically different between the delayed feedback condition $(\mathrm{M}=72.51, \mathrm{SD}=23.28)$ and the baseline condition $(\mathrm{M}=77.50$, $\mathrm{SD}=43.34), t(4)=-0.50, \mathrm{p}=.64, \eta^{2}=0.06$, suggesting no change in task difficulty.

\section{Discussion}

Stetson et al. (2006) have shown a reversal effect in the subjective order of simultaneous action and sensation following an adaptation phase, in which participants were adapted to a delayed sensory feedback regime. Here, we implemented this visuo-motor recalibration paradigm into a single-change color-motion asynchrony task and provided evidence that the visuo-motor recalibration may reduce the asynchrony in the binding of visual features in the presence of an action-sensory outcome association. Our results showed that:

(1) As well as a first-order change as has been previously shown by Stetson et al. (2006), the temporal recalibration due to a delayed sensory feedback in response to a goal-directed voluntary action can also be induced using a second-order change. (Experiment 1).

(2) Adaptation to a delay in the relative timing between a self-initiated manual action and the related motion change during the formation of an action-sensory outcome association reduces the color-motion asynchrony in the following test trials, where the motion change is made contingent upon a key press (Experiment 2A).

(3) Visuo-motor temporal recalibration generated using a single visual feature change (motion direction) being contingent upon a motor act is transferable to a multi-feature stimuli set (i.e., chromatic dots changing motion direction) in a color-motion correspondence task (Experiment 2A), supporting the earlier findings on the transfer of adaptation across different sensory modalities (Heron et al., 2009; Sugano et al., 2010).

(4) The reduction in the color-motion asynchrony in the visuo-motor condition is significantly larger than that in the passive visual condition, suggesting that the effect is not a result of a pure visual adaptation (Experiment 2B) but rather, requires the execution of a motor action and possibly, the related action-outcome prediction.

(5) The reduction in the color-motion asynchrony effect size following a motor-sensory recalibration (Experiment 2A) is larger than the bias in the perceived onset of direction change relative to that of the color (Experiment 3). Thus, the CMA results may not be explained within the context of a single timeline idea, where the onset positions of the sensory events are perceptually shifted.
It has been suggested that CMA is the result of a neurophysiological effect called opposite-motion inhibition (Snowden et al., 1991), which introduces a delay in forming a new motion-defined surface representation at the area MT + (Arnold \& Clifford, 2002; Bedell et al., 2003; Clifford et al., 2004, 2010), particularly because the largest asynchrony occurs for changes in opponent directions (180 deg apart) (Arnold \& Clifford, 2002). Clifford et al. (2004) further demonstrated that the percept of asynchrony between color and motion is reduced substantially in a paradigm, where arrays of dots move in opposite directions as sequentially organized thin stripes, rather than as homogenous blocks. This facilitation of perceptual binding in the presence of motion transparency was linked to the feedback connections from the area MT + to the cortical areas with higher spatial resolution (i.e. primary visual cortex) (Clifford, 2010), where neural activity has been previously shown to be involved in perceptual binding (Hochstein \& Ahissar, 2002). In this account (Clifford, 2010), local motion signals processed in the primary visual cortex are sent to the motion-sensitive extrastriate areas to be integrated into a surface representation. These signals are then sent back to early cortical areas, where neurons are reciprocally associated with the extrastriate representations of the motion-defined surfaces. Finally, neurons in primary visual cortex associated with the surface feed their signals into the higher-level color processing areas, where surface color is determined. In this context, directional inhibition (Snowden et al., 1991) slows down the formation of a new motion-defined surface representation, introducing a temporal delay. In the case of motion transparency, on the other hand, motion representation is maintained for both transparent motion-defined surfaces, thus, color and motion can be bound temporally accurately (Clifford et al., 2004). In the context of this opposite-motion inhibition account, what we suggest here is that a sensory prediction signal (i.e. a particular motion direction) generated on the basis of a motor-sensory association might play a similar role to motion transparency in silencing the opposite-direction inhibition and thus, facilitating the binding of concurrent motion and color signals.

One explanation as to how action recalibrates sensory representations of temporal events has been provided within the context of a predictive coding account (Shi \& Burr, 2016), where a prior about the temporal representations of multimodal events (i.e. action and its sensory outcome) are updated dynamically through the delay adaptation trials. In the following test blocks, when the delay is removed, the actual current sensory timing does not overlap with the prior, thus, yielding a prediction error (i.e. reversal in the subjective order of action and sensory order). On this account, the brain constantly tries to minimize prediction errors by updating the internal temporal prior based on Bayesian inference. Such predictive coding has been suggested to account for neural activity in the visual cortex during both freeviewing (Gallant, Connor, \& Van Essen, 1998) and extra-classical receptive field effects (Rao \& Ballard, 1999). Thus, here, what we claim is that perceptual feature binding may also have a similar mechanism, which involves inferential processes through feedback connections. Such Bayesian accounts of CMA are alternatives to brain-time accounts as they explain the phenomena in terms of features of the represented content rather than in terms of temporal properties of the vehicles of representation. In a paradigm, where a key press was either associated with a visual effect or not, Hughes and Waszak (2011) proposed that one physiological measure of the prediction of voluntary action effects is the lateralized difference in readiness potentials in action-predicts-avisual-outcome versus action-only trials, which has been suggested to be generated in the primary motor cortex (Coles, 1989; Leuthold \& Jentzsch, 2002). During a visually guided motor action, populations of neurons in the primary motor cortex have also been shown to display a co-activity with those in the area MT + , which particularly code for similar directions of movement (Kruse et al., 2002). Using magnetoencephalography (MEG) in combination with functional MRI (fMRI), Cai, Ogawa, Kochiyama, Tanaka, and Imamizu (2018) have investigated the neural correlates of changes following motor-sensory 
recalibration and demonstrated that the readiness potentials in the motor system, particularly in the parietal regions get delayed, whereas visually evoked potentials in the extrastriate areas of the occipital regions significantly shift earlier due to the delay adaptation. This implies that delay adaptation can speed up neural processes in the visual cortex, probably via predictive signals from the motor-related areas to motionsensitive extrastriate areas (e.g. MT+), and reduce the latency caused by opposite-motion inhibition (Snowden et al., 1991). In fact, Sugano, Keetels, and Vroomen (2017) have demonstrated that motor-sensory delay adaptation fastens the reaction time to auditory stimuli, speedingup auditory latencies in the early auditory cortex, but does not induce similar shifts in the reaction to visual stimuli, which provides further evidence that the shift in visually evoked potentials in Cai et al. (2018) is most probably linked to the feedback signals from the parietal regions to extrastriate occipital areas, rather than a speed-up in the neural processes in the early visual cortex. Given this link between the area MT + and the primary motor cortex, the activity of which has been linked to action-induced sensory prediction, we propose that as well as motion transparency (Clifford et al., 2004), motor-sensory recalibration, originally demonstrated by Stetson et al. (2006), might also exert its influences during the formation of surface representations in the global motion processing visual cortex.

Forward models of motor control (Miall \& Wolpert, 1996) posit an imitation of the causal flow triggered by an action, where a copy of the motor command ("efference copy") is used to predict the sensory outcome or anticipate the motor antecedent of the ongoing action. The actual sensations of the self-generated actions are then compared to the predicted sensory outcomes, and a match between the two results in sensory attenuation. We think that the reason why we observed a larger color-motion asynchrony in the baseline condition of Experiment $2 \mathrm{~A}$ $(\mathrm{M}=62.22 \mathrm{~ms})$ compared to that of Experiment $2 \mathrm{~B}(\mathrm{M}=36.20 \mathrm{~ms})$ may be the presence of sensory attenuation in Experiment 2A, as well as the opposite-motion inhibition. In Experiment 2A, the sensory outcome is contingent upon the key press and presented without a delay during both the baseline adaptation and the test trials, forming an anticipation signal which would suppress the sensory outcome (in this case, the change of motion direction). This signal, on the other hand, is absent in Experiment 2B, as there has been no key press during the test trials.

It has been demonstrated that the efference copy (corollary discharge) does not only signal a prediction with respect to the content of the sensory outcome, but also carry temporal information as to when to estimate the associated sensations (Blakemore, Frith, \& Wolpert, 1999; Elijah, Le Pelley, \& Whitford, 2016). In a recent study, using electroencephalography, Elijah et al. (2016) showed that N1 amplitudes to delayed tones, being significantly larger in a motor action - sensory outcome (button-press-for-tone) association task in the pre-training phase, get significantly reduced following a delayed-adaptation training phase, where sensory outcome is presented with a delay of $100 \mathrm{~ms}$. N1 component of event-related potentials are known to reflect the physical (Mulert et al., 2005; Simmons, Nathan, Berger, \& Allen, 2011) intensity of the sensory stimulus. Compatibly, self-generated sensory stimuli were shown to elicit smaller N1 amplitudes than the same stimulus presented externally (Baess, Horváth, Jacobsen, \& Schröger, 2011; Bäß, Jacobsen, \& Schröger, 2008), implying that N1 amplitude may also be an index of perceived intensity. That the $\mathrm{N} 1$ amplitude is reduced not immediately after but following the trained delay in the post-training phase of Elijah et al. study suggests that the behavioral training to a delay of sensory outcome may modify the neural expectations regarding the timing of sensations contingent upon the actions. In this context, we propose that during the delayed adaptation phase of our paradigm, the anticipated timing of the sensory attenuation is shifted to a later temporal window. Because the sensory outcome is presented without a delay in the test trial, however, the anticipated time of the sensory outcome does not overlap with the timing of the current sensory signal, thus the effect of sensory attenuation is avoided. As a result, the neural representation of the new motion surface is disentangled from the inhibitory influences, and the color-motion asynchrony gets reduced. Thus, in this case, motor-act-sensory-outcome delay adaptation is not only important in forming action-sensory outcome associations but may also act as a binding factor between the two perceptual features of a sensory event.

In the well-known intentional binding effect, volitional actions are perceived as temporally shifted toward their sensory effects (Haggard, 2005; Haggard, Clark, \& Kalogeras, 2002). In a typical paradigm, participants are asked to indicate the perceived time of their voluntary actions, or of a sensory outcome (i.e. a beep) presented with a brief delay. Perceptual judgments in these experimental blocks are then compared to a baseline, where judgments are made on an action without a sensory outcome (Haggard et al., 2002). Results demonstrate that the perceived time between the volitional action and the subsequently presented sensory event is compressed within the context of action-sensory outcome association. That the effect of CMA did not differ from the baseline in our visual temporal ordering task, where participants judged the temporal order of motion direction and color change following a visuo-motor recalibration, however, demonstrates that the relative perceived time point, when direction change takes place in relation to color change was judged similarly in the baseline and delay adaptation conditions. Interestingly, we observed a statistically non-significant trend in the overall data such that direction change was judged to occur earlier than the color change, which might be linked to the intentional binding effect exerting similar influences in the baseline and delay adaptation conditions. Color-motion asynchrony being absent in Experiment 3 is consistent with the previous report (Bedell et al., 2003), where CMA was shown to be task-dependent such that asynchrony was apparent in the feature correspondence task but disappeared in the temporal ordering task. Because in our paradigm, all local motion signals were as same as the global motion signal, what we suggest is that the temporal order judgments in Experiment 3 were made at the level of representations formed in the primary visual cortex, whereas color correspondence judgments in Experiment 2A required the formation of surface representation at level of MT + , the activity of which was modulated by the predictive influences from the parietal cortex. It is at the level of MT + that we think the motor-sensory recalibration has an effect on the color-motion asynchrony. Altogether these findings demonstrate that the detection of motion change does not necessarily entail a correct phase association between the color and the direction, which provides further evidence that the temporal association is formed between the key press and the formation of the surface representation defined by the second direction, rather than the time point at which the direction changes.

In a temporal marker account of CMA, Nishida and Johnston (2002) proposed that the asynchrony occurs because participants cannot match a second-order change (i.e. motion direction) to a first-order change (i.e. color) at high-frequency alterations, thus, what is marked as a salient stimulus transition is a change in position (as a first-order feature) rather than a change in motion direction. This account presumes a repeated and rapid alternation of motion direction, which results in an erroneous matching between higher-level correspondence of temporal markers. Linares and López-Moliner (2006), however, have later demonstrated that the asynchrony is still observed using a single direction change paradigm, where the repeated oscillation is absent, contradicting the time marker explanation. Here, using a similar paradigm to that of Linares and López-Moliner (2006), where the direction change was either contingent upon a self-initiated action or purely visual following a visuo-motor adaptation, we also found a significant CMA effect, providing further evidence that the repeated oscillation of the stimuli is not a necessary condition to induce color-motion asynchrony.

It has been show that intention is an essential component of the motor-sensory temporal recalibration, as the effect is not simply driven by a temporal relationship between tactile perception and auditory effect, but the intentional act of pressing a button (Stetson et al., 2006). Arnold, Nancarrow, and Yarrow (2012) have demonstrated that what is 
crucial for motor-sensory recalibration is the temporal relationship between the termination of an action and the subsequent sensory output, rather than the timing of decision to act or motor planning activity, pointing out to the importance of "the sensation of having acted" in determining the magnitude of the effect. Agency or authorship is defined by Ebert and Wegner (2010) as "perceiving one's own action as the cause of the subsequent event". During the delayed feedback adaptation in response to the self-generated voluntary action, we manipulated the temporal structure of events, which by habitude were expected to be temporally closer in time. That we only observed a perceptual feature binding effect in the visuo-motor condition, where participants were not only passive observers but rather active agents, we conclude that the observed results can be ascribed to an actionoutcome pairing acquired during the delay adaptation as result of a causality-based agency. In fact, recent evidence demonstrates that not only the perceived temporal order of an action and its sensory outcome, but perceived agency is also subject to recalibration by the motor sensory delay adaptation training (Haering \& Kiesel, 2015, 2016; Timm, Schönwiesner, SanMiguel, \& Schröger, 2014). In this context, we suggest that this perceived causality may have a certain role in regulating the temporal expectancies for the outcome of the executed actions.

In a paradigm, where they adapted participants to asynchronous sensory inputs in a grating stimulus setup oscillating both in color (red/ green) and in motion direction (left/right), Arnold and Yarrow (2011) have demonstrated that temporally offset color and motion direction can be reported as synchronous in the test trials following the shift in the Point of Subjective Simultaneity towards the adapted direction. This demonstrates that simultaneity judgments between the two withinmodal features (e.g. color and motion direction in the visual modality) can be subject to sensory temporal recalibration. Motor-sensory recalibration as we show here, on the other hand, seem to modulate colormotion asynchrony in the correspondence, rather than in the temporal order judgment task, implying that the two effects possibly have different neural underpinnings. In fact, as we suggest above, whereas the temporal association in our paradigm is formed between the key press and the formation of the surface representation defined by the second direction, rather than the time point at which the direction changes, the effect in the study of Arnold and Yarrow (2011) seems to be caused by a change in the apparent timing of color and direction changes. In this context, motor-sensory delay adaptation, where intentionality does play an essential role, is different than adaptation to within- or crossmodal delays between passively sensed features (Rohde \& Ernst, 2016).

In sum, voluntary causal action has an influence on perceptual feature binding. Visuo-motor recalibration does not act by shifting the onset of direction change relative to that of the color, which together may imply that the reduction in the CMA effect size is due to the actionoutcome prediction within the perspective of predictive coding. These results seem to be consistent with the recent neuroscientific accounts of receptive field properties in the visual cortex.

\section{CRediT authorship contribution statement}

Inci Ayhan: Funding acquisition, resources, conceptualization, methodology, formal analysis, software, supervision, writing - original draft, writing - review \& editing. Melisa Kurtcan: Conceptualization, investigation, data curation, formal analysis, visualization, writing original draft. Lucas Thorpe: Conceptualization, investigation, resources, supervision, writing - original draft.

\section{Acknowledgments}

Our research is funded by Bogazici University Scientific Research Projects, Project No: 9248 awarded to I.A. We are grateful to David Burr and Esra Mungan for their comments and discussions concerning this work.

\section{Appendix A. Supplementary data}

Supplementary data to this article can be found online at https:// doi.org/10.1016/j.visres.2020.09.001.

\section{References}

Arnold, D. H., \& Clifford, C. W. (2002). Determinants of asynchronous processing in vision. Proceedings of the Royal Society of London. Series B: Biological Sciences, 269(1491), 579-583.

Arnold, D. H., Nancarrow, K., \& Yarrow, K. (2012). The critical events for motor-sensory temporal recalibration. Frontiers in Human Neuroscience, 6, 235.

Arnold, D. H., \& Yarrow, K. (2011). Temporal recalibration of vision. Proceedings of the Royal Society B: Biological Sciences, 278(1705), 535-538.

Baess, P., Horváth, J., Jacobsen, T., \& Schröger, E. (2011). Selective suppression of selfinitiated sounds in an auditory stream: An ERP study. Psychophysiology, 48(9), $1276-1283$.

Bartels, A., \& Zeki, S. (1998). The theory of multistage integration in the visual brain. Proceedings of the Royal Society of London. Series B: Biological Sciences, 265(1412), 2327-2332.

Bäß, P., Jacobsen, T., \& Schröger, E. (2008). Suppression of the auditory N1 event-related potential component with unpredictable self-initiated tones: Evidence for internal forward models with dynamic stimulation. International Journal of Psychophysiology, $70(2), 137-143$.

Bedell, H. E., Chung, S. T. L., Ogmen, H., \& Patel, S. S. (2003). Color and motion: Which is the tortoise and which is the hare? Vision Research, 43(23), 2403-2412.

Blakemore, S.-J., Frith, C. D., \& Wolpert, D. M. (1999). Spatio-temporal prediction modulates the perception of self-produced stimuli. Journal of Cognitive Neuroscience, 11(5), 551-559.

Cai, C., Ogawa, K., Kochiyama, T., Tanaka, H., \& Imamizu, H. (2018). Temporal recalibration of motor and visual potentials in lag adaptation in voluntary movement. NeuroImage, 172, 654-662.

Cavanagh, P., MacLeod, D. I. A., \& Anstis, S. M. (1987). Equiluminance: Spatial and temporal factors and the contribution of blue-sensitive cones. Journal of the Optical Society of America A, 4(8), 1428-1438.

Clifford, C. W. G., Spehar, B., \& Pearson, J. (2004). Motion transparency promotes synchronous perceptual binding. Vision Research, 44(26), 3073-3080.

Clifford, C. W. G. (2010). Dynamics of visual feature binding. Space and Time in Perception and Action, 199.

Colby, C. L., Duhamel, J. R., \& Goldberg, M. E. (1989). Visual response properties and attentional modulation of neurons in the ventral intraparietal area (VIP) in the alert monkey. In Soc Neurosci Abstr (Vol. 15, p. 162).

Coles, M. G. H. (1989). Modern mind-brain reading: psychophysiology, physiology, and cognition. Psychophysiology, 26(3), 251-269.

Derrington, A. M., Krauskopf, J., \& Lennie, P. (1984). Chromatic mechanisms in lateral geniculate nucleus of macaque. The Journal of Physiology, 357(1), 241-265.

Duhamel, J. R., Colby, C. L., \& Goldberg, M. E. (1989). Congruent visual and somatosensory response properties of neurons in the ventral intraparietal area (VIP) in the alert monkey. In Soc Neurosci Abstr (Vol. 15, p. 162)

Ebert, J. P., \& Wegner, D. M. (2010). Time warp: Authorship shapes the perceived timing of actions and events. Consciousness and Cognition, 19(1), 481-489.

Elijah, R. B., Le Pelley, M. E., \& Whitford, T. J. (2016). Modifying temporal expectations: Changing cortical responsivity to delayed self-initiated sensations with training. Biological Psychology, 120, 88-95.

Gallant, J. L., Connor, C. E., \& Van Essen, D. C. (1998). Neural activity in areas V1, V2 and V4 during free viewing of natural scenes compared to controlled viewing. NeuroReport, 9(9), 2153-2158.

Haering, C., \& Kiesel, A. (2015). Was it me when it happened too early? Experience of delayed effects shapes sense of agency. Cognition, 136, 38-42.

Haering, C., \& Kiesel, A. (2016). Time perception and the experience of agency. Psychological Research, 80(2), 286-297.

Haggard, P., Clark, S., \& Kalogeras, J. (2002). Voluntary action and conscious awareness. Nature Neuroscience, 5(4), 382-385.

Haggard, P. (2005). Conscious intention and motor cognition. Trends in Cognitive Sciences, 9(6), 290-295.

Heron, J., Hanson, J. V., \& Whitaker, D. (2009). Effect before cause: supramodal recalibration of sensorimotor timing. PLoS One, 4(11), Article e7681.

Hochstein, S., \& Ahissar, M. (2002). View from the top: Hierarchies and reverse hierarchies in the visual system. Neuron, 36(5), 791-804.

Hughes, G., \& Waszak, F. (2011). ERP correlates of action effect prediction and visual sensory attenuation in voluntary action. NeuroImage, 56(3), 1632-1640.

Kruse, W., Dannenberg, S., Kleiser, R., \& Hoffmann, K. P. (2002). Temporal relation of population activity in visual areas MT/MST and in primary motor cortex during visually guided tracking movements. Cerebral Cortex, 12(5), 466-476.

Leuthold, H., \& Jentzsch, I. (2002). Distinguishing neural sources of movement preparation and execution. Biological Psychology, 60(2-3), 173-198.

Linares, D., \& López-Moliner, J. (2006). Perceptual asynchrony between color and motion with a single direction change. Journal of Vision, 6(9), 10.

Livingstone, M., \& Hubel, D. (1988). Segregation of form, color, movement, and depth: Anatomy, physiology, and perception. Science, 240(4853), 740-749.

Maunsell, J. H., \& van Essen, D. C. (1983). The connections of the middle temporal visual area (MT) and their relationship to a cortical hierarchy in the macaque monkey Journal of Neuroscience, 3(12), 2563-2586.

Miall, R. C., \& Wolpert, D. M. (1996). Forward models for physiological motor control. 
Neural Networks, 9(8), 1265-1279.

Moutoussis, K., \& Zeki, S. (1997). A direct demonstration of perceptual asynchrony in vision. Proceedings of the Royal Society of London. Series B: Biological Sciences, 264(1380), 393-399.

Mulert, C., Jäger, L., Propp, S., Karch, S., Störmann, S., Pogarell, O., Möller, H.-J., Juckel, G., ... Hegerl, U. (2005). Sound level dependence of the primary auditory cortex: Simultaneous measurement with 61-channel EEG and fMRI. NeuroImage, 28(1), 49-58.

Nishida, S., \& Johnston, A. (2002). Marker correspondence, not processing latency, determines temporal binding of visual attributes. Current Biology, 12(5), 359-368.

Rao, R. P. N., \& Ballard, D. H. (1999). Predictive coding in the visual cortex: A functional interpretation of some extra-classical receptive-field effects. Journal of Neuroscience, 2(1), 79-87.

Rohde, M., \& Ernst, M. O. (2016). Time, agency, and sensory feedback delays during action. Current Opinion in Behavioral Sciences, 8, 193-199.

Rohde, M., Greiner, L., \& Ernst, M. O. (2014). Asymmetries in visuomotor recalibration of time perception: Does causal binding distort the window of integration? Acta Psychologica, 147, 127-135.

Shi, Z., \& Burr, D. (2016). Predictive coding of multisensory timing. Current Opinion in Behavioral Sciences, 8, 200-206.

Shipp, S., \& Zeki, S. (1985). Segregation of pathways leading from area V2 to areas V4 and V5 of macaque monkey visual cortex. Nature, 315(6017), 322-324.

Simmons, J. G., Nathan, P. J., Berger, G., \& Allen, N. B. (2011). Chronic modulation of serotonergic neurotransmission with sertraline attenuates the loudness dependence of the auditory evoked potential in healthy participants. Psychopharmacology, 217(1), 101-110.

Snowden, R. J., Treue, S., Erickson, R. G., \& Andersen, R. A. (1991). The response of area MT and V1 neurons to transparent motion. Journal of Neuroscience, 11(9), 2768-2785.

Stetson, C., Cui, X., Montague, P. R., \& Eagleman, D. M. (2006). Motor-sensory recalibration leads to an illusory reversal of action and sensation. Neuron, 51(5), 651-659.

Sugano, Y., Keetels, M., \& Vroomen, J. (2010). Adaptation to motor-visual and motorauditory temporal lags transfer across modalities. Experimental Brain Research 201(3), 393-399.

Sugano, Y., Keetels, M., \& Vroomen, J. (2017). Audio-motor but not visuo-motor temporal recalibration speeds up sensory processing. PloS One, 12(12), Article e0189242.

Timm, J., Schönwiesner, M., SanMiguel, I., \& Schröger, E. (2014). Sensation of agency and perception of temporal order. Consciousness and Cognition, 23, 42-52.

Ungerleider, L. G., \& Desimone, R. (1986). Cortical connections of visual area MT in the macaque. Journal of Comparative Neurology, 248(2), 190-222.

Watson, A. B. (1979). Probability summation over time. Vision Research, 19(5), 515-522. Yarrow, K., Sverdrup-Stueland, I., Roseboom, W., \& Arnold, D. H. (2013). Sensorimotor temporal recalibration within and across limbs. Journal of Experimental Psychology: Human Perception and Performance, 39(6), 1678-1689.

Zeki, S., \& Bartels, A. (1998). The asynchrony of consciousness. Proceedings of the Royal Society of London. Series B: Biological Sciences, 265(1405), 1583-1585. 